\title{
Somogy megye levéldarázs-alkatúinak katalógusa (Hymenoptera, Symphyta)
}

\author{
Haris ATtILA
}

\begin{abstract}
HARIS A.: Catalogue of the sawfly fauna of Somogy county (Hymenoptera: Symphyta)
Abstract: 220 Symphyta species are listed from Somogy county. Phyllocolpa leucaspis (Tischbein, 1846) and Pristiphora bifida Hellén, 1947 are new species for Hungarian fauna. Further rare species are Athalia paradoxa Konow, 1886; Dolerus megapterus Cameron, 1881; Dolerus quadrinotatus Bíró, 1884; Pamphilius marginatus (Lepeletier, 1823); Pama tenella (Klug, 1816); Strongylogaster xanthocera (Stephens, 1835); Tomostethus funereus (Klug, 1816); Tremex alchymista Mocsáry, 1836 and Trichosoma vitellinae (Linné, 1761)
\end{abstract}

\section{Bevezetés}

A Symphytakra vonatkozó elsố Somogy megyei adataink MoCSÁRY SÁNDOR (1900) által szerkesztett Fauna Regni Hungariae kötetben találhatók. Ez a munka a kor magyar faunisztikájának összegzô múve. Az azóta eltelt 100 év során intenzív faunisztikai gyújitések indultak meg az országban, így Somogy megyében is, ennek ellenére a hymenopterák ezen csoportja a kevésbé kutatottak közé tartozik. Somogy megyére vonatkozóan csupán két település és környékéról vannak viszonylag részletes faunisztikai adataink (ZOMBORI, 1985, HARIS, 1998). Ezeken túlmenően a csoportról eddig megjelent faunakötetek tartalmaznak adatokat Somogy megyéból, túlnyomórészt a Magyar Természettudományi Múzeum anyagára alapozva.

MOCSÁRY (1900) 5 fajt említ a megye területérôl. Ezek közül egy faj különösen kiemelésre méltó: Trichosoma vitellinae (Linné, 1761). A genus fajai északi elterjedésúek, ritkák. Magyarország elterjedésük déli határán fekszik. A faj azóta sem került elổ Somogyból. A csurgói lelôhelyen kívül csupán I magyarországi adata ismeretes (Bakonybél).

A megyén belül viszonylag intenzíven kutatott terület egyedül Kaposmérô és környéke, néhány fajt ZOMBORI (1985) publikált a Barcsi Ósborókás területéról. További szórvány adatokkal rendelkezünk Kaposvár, Zamárdi, Látrány településekről.

A következö listán megadjuk a szakirodalomból, közgyújteményekből (Kaposvár, Budapest) ismert fajok jegyzékét.

Az itt szereplő 220 faj a teljes magyar faunának mintegy harmada. A magyarországi fajok becsült száma 600 fajra tehetô.

$\mathrm{Az}$ idézett szakirodalom eredeti névhasználata ebben a listában természetesen nem mindig található meg. A megjelenésük óta bevezetett nevezéktani változásokat többségében itt is alkalmaztuk, de csak ott, ahol ezeket követendőnek ítéltük meg.

A magyar fauna rendszertanilag 6 családsorozatra és 12 családra oszlik. A fajok rendszertani megoszlását a lista végén az értékelés fejezetben táblázatos formában közöljük. 


\section{Somogyból ismert fajok jegyzéke}

A listában használt rövidítések:

MTM - Magyar Természettudományi Múzeum gyưjteménye

SMM - Somogy Megyei Múzeum gyújteménye

\section{SYMPHYTA}

\section{Pamphilidae}

Acantholyda hieroglyphica (Christ, 1791)

Zombori, 1985

Cephalcia abietis (Linné, 1758)

Móczár et Zombori, 1973

Neurotoma nemoralis (Linné, 1758)

Zamárdi felsô, MTM

Pamphilius marginatus (Lepeletier, 1823)

Haris, 1998

Pamphilius sylvaticus (Linné, 1758)

Lad, SMM

Pamphilius vafer (Linné, 1767)

Látrány, SMM

Megalodontidae

Melanopus fabricii (Leach, 1817)

Mocsáry, 1900

Megalodontes plagiocephalus (Fabricius, 1804)

Látrány, SMM

Megalodontes flavicornis (Klug, 1824)

Csököly, SMM

Megalodontes cephalotes (Fabricius, 1781)

Pusztaszemes, SMM

\section{Xiphidridae}

Xiphidria camelus (Linné, 1758)

Haris, 1998, Zombori, 1985

Xiphidria prolongata (Geoffroy, 1785)

Zamárdi felsó, MTM

\section{Siricidae}

Tremex alchymista Mocsáry, 1836

Haris, 1998

Uroceros gigas (Linné, 1758)

Haris, 1998

Orussidae

Orussus abietinus (Scopoli, 1.763)

Darány, Barcs, SMM

\section{Cephidae}

Calameuta filiformis (Eversmann, 1847)

Vörs, MTM

Calameuta haemorrhoidalis (Fabricius, 1781)

Vörs, MTM, Somogyaszaló, Nagybajom, SMM

Calameuta pallipes (Klug, 1803)

Zombori, 1985
Calameuta punctata (Klug, 1803)

Látrány, SMM

Cephus brachycerus C. G. Thomson, 187 I

Haris, 1998

Cephus cultratus Eversmann, 1847

Kis-Balaton, MTM

Cephus nigrinus C. G. Thomson, 1871

Haris, 1998

Cephus pygmaeus (Linné, 1767)

Haris, 1998, Zombori, 1985

Cephus runcator Konow, 1896

Zombori, 1985

Janus compressus (Fabricius, 1793)

Móczár et Zombori, 1973

Argidae

Aprosthema bifida (Klug, 1834)

Móczár et Zombori, 1973

Arge berberidis Schrank, 1802

Haris, 1998

Arge ciliaris (Linné, 1767)

Móczár et Zombori, 1973

Arge cyanocroeca (Förster, 1871)

Haris, 1998, Zombori, 1985

Arge enodis (Linné, 1767)

Haris, 1998

Arge gracilicomis (Klug, 1814)

Haris, 1998, Zombori, 1985

Arge melanochroa (Gmelin, 1790)

Haris, 1998

Arge ochropus (Gmelin, 1790)

Fonyódliget, MTM

Arge nigripes (Retzius, 1783)

Haris, 1998

Arge pagana (Panzer, 1798)

Haris, 1998, Zombori, 1985

Arge rustica (Linné, 1758)

Haris, 1998, Zombori, 1985

Sterictophora furcata (Villers, 1789)

Vörs, MTM

\section{Cimbicidae}

Abia fulgens Zaddach, 1863

Móczár et Zombori, 1973

Abia nitens (Linné, 1758)

Haris, 1998

Abia sericea (Linné, 1767)

Haris, 1998

Cimbex connata (Schrank, 1776) 
Hosszúvíz, SMM

Cimbex femorata (Linné, 1758)

Haris, 1998

Corynis crassicomis (Rossi, 1790)

Kis-Balaton, Diás sziget, MTM

Corynis obscura (Fabricius, 1775)

Haris, 1998

Palaeocimbex quadrimaculata (O.F. Müller, 1766)

Haris, 1998, Zombori, 1985

Pseudocavellaria amerinae (Linné, 1758)

Zamárdi felsó, MTM

Trichosoma vitellinae (Linné, 1761)

Mocsáry, 1900

\section{Diprionidae}

Diprion pini (Linné, 1758)

Haris, 1998

Gilpina frutetorum (Fabricius, 1793)

Móczár et Zombori, 1973

Gilpina laricis (Jurine, 1807)

Haris, 1998

Gilpina virens (Klug, 1812)

Móczár et Zombori, 1973, Zombori, 1985

Monoctenus obscuoatus ssp. intermedius Zombori, 1975

Zombori, 1985

\section{Tenthredinidae}

\section{Allantinae}

Allantus calceatus (Klug, 1818)

Haris, 1998 mint Emphytus calceatus (Klug, I818)

Allantus cinctus (Linné, 1758)

Kaposvár, MTM

Allantus didymus (Klug, 1818)

Vízvár, SMM

Allantus melanarius (Klug, 1818)

Haris, 1998 mint Emplytus melanarius (Klug, 181 8)

Allantus truncatus (Klug, 1818)

Zombori, 1982, Zombori, 1985 mint Emphytus truncatus (Klug, 1818)

Ametastegia carpini (Hartig, 1837)

Nágocs, SMM

Ametastegia equiseti (Fallén, 1808)

Haris, 1998, Zombori, 1985

Ametastegia pallipes (Spinola, 1808)

Haris, 1998

Ametastegia tener (Fallén, 1808)

Haris, 1998, Zombori, 1985 mint Protemphytus tener (Fallén, 1808)

Apethymus serotinus (O. F. Müller, I776)

Zombori, 1985 mint Aphetymus braccatus

(Gmelin, 1790)

Athalia bicolor (Lepeletier, 1823)

Haris, 1998

Athalia circularis (Klug, 1815)

Haris, 1998, Zombori, 1985
Athalia cornubiae Benson, 1931

Haris, 1998, Zombori, 1985

Athalia cordata Lepeletier, 1823

Haris, 1998, Zombori, 1985

Athalia glabricollis Thomson, 1870

Zombori, 1985

Athalia liberta (Klug, 1815)

Haris, 1998

Athalia lugens (Klug, 1815)

Zombori, 1982

Athalia paradoxa Konow, 1886

Zombori, 1982

Athalia rosae (Linné, 1758)

Haris, 1998, Zombori, 1985

Caliroa cerasi (Linné, 1758)

Haris, 1998

Caliroa varipes (KJug, 1816)

Látrány, SMM

Empria klugii (Stephens, 1835)

Haris, 1998

Empria liturata (Gmelin, 1790)

Látrány, SMM

Empria pumila (Konow, 1896)

Haris, 1998

Empria tridens (Konow, 1896)

Látrány, SMM

Eriocampa ovata (Linné, 1761)

Haris, 1998

Monostegia abdominalis (Fabricius, 1798)

Haris, 1998

Monostegia cingulata (Konow, 1891)

Haris, 1998

Monsoma pulveratum (Retzius, 1783)

Látrány, SMM

Taxonus agrorum (Fallén, 1808)

Haris, 1998, Zombori, 1985

Taxonus sticticus (Klug, 1817)

Haris, 1998

Selandrinae

Aneugmenus coronatus (Klug, 1818)

Zombori, 1982, Zombori, 1985

Aneugmenus padi (Linné, 1761)

Mocsáry, 1900 mint Selandria stramineipes Klug, 1814

Nesoselandria morio (Fabricius, 1781)

Haris, 1998

Selandria serva (Fabricius, 1793)

Haris, 1998, Zombori, 1985

Strongylogaster lineata (Christ, 1791)

Haris, 1998

Strongylogaster xanthocera (Stephens, 1835)

Zombori, I 982, Zombori, 1985

Dolerinae

Dolerus aericeps (C. G. Thomson, 1871) 
Haris, 1998

Dolerus anthracinus (Klug, 1818)

Haris, 1998, Zombori, 1985

Dolerus asper Zaddach, 1859

Haris, 1998

Dolerus bimaculatus (Geoffroy, 1785)

Haris, 1998, Mocsáry, 1900 mint Dolerus analis

Konow

Dolerus germanicus (Fabricius, 1775)

Haris, 1998

Dolerus gonager (Fabricius, 1781)

Haris, 1998

Dolerus haematodes (Schrank, 1781)

Kis-Balaton, Lebujpuszta, MTM

Dolerus megapterus Cameron, 1881

Kis-Balaton, MTM

Dolerus niger (Linné, 1767)

Haris, 1998

Dolerus nigratus (O. F. Müller, 1776)

Haris, 1998, Zombori, 1985

Dolerus nigrominutus Haris, 1998

Látrány, SMM

Dolerus nitens Zaddach, 1859

Haris, 1998 Dolenus possilensis Cameron missid.,

Zombori, 1985

Dolerus picipes (Klug, 1818)

Haris, 1998, Zombori, 1985

Dolerus puncticollis C. G. Thomson, 1871

Haris, 1998

Dolerus sanguinicollis (Klug, 1818)

Kaposfó, MTM

Dolerus quadrinotatus Bíró, 1884

Látrány, SMM

Dolerus thoracicus (Fallén, 1808)

Zombori, 1982, Zombori, 1985

Dolerus triplicatus (Klug, 1818)

Haris, 1998

Dolerus vestigialis (Klug, 1818)

Haris, 1998

\section{Blennocampinae}

Atomostethus ephippium (Panzer, 1798)

Haris, 1998, Zombori, 1985

Blennocampa pusilla (Klug, 1816)

Haris, 1998, Zombori, 1985

Cladardis elongatula (Klug, 1817)

Haris, 1998

Claremontia alternipes (Klug, 1816)

Haris, 1998

Claremontia confusa (Konow, 1886)

Kis-Balaton, Zala part, MTM

Claremontia tenuicornis (Klug, 1816)

Haris, 1998

Eutomostethus luteiventris (Klug, 1816)

Haris, 1998
Fenella nigrita Westwood, 1840

Zombori, 1990

Fenusa pusilla (Lepeletier, 1823)

Haris, 1998

Halidamia affinis (Fallén, 1807)

Haris, 1998

Heterarthrus vagans (Fallén, 1808)

Haris, 1998

Metallus pumilus (Klug, 1814)

Haris, 1998

Monophadnoides rubi (Harris, 1845)

Haris, 1998

Monphadnus pallescens (Gmelin, 1790)

Haris, 1998

Monphadnus spinolae (Klug, 1816)

Haris, 1998

Pareophora pruni (Linné, 1758)

Zamárdi-felsô, MTM

Parna tenella (Klug, 1816)

Haris, 1998

Periclista albiventris (Klug, 1816)

Látrány, SMM

Stethomostus fulliginosus (Schrank, 1781)

Haris, 1998, Zombori, 1985

Tomostethus funereus (Klug, 1816)

Zombori, 1990, Zombori, 1985 mint

Stethomostus funereus (Klug, 1814)

\section{Tenthredininae}

Aglaostigma aucupariae (Klug, 1817)

Haris, 1998, Zombori, 1985

Aglaostigma fulvipes (Scopoli, 1763)

Haris, 1998, Zombori, 1985

Aglaostigma nivosa (Klug, 1817)

Haris, 1998 mint Tenthredopsis nivosa (Klug,

1817)

Macrophya albicincta (Schrank, 1776)

Haris, 1998, Mocsáry, 1900

Macrophya annulata (Geoffroy, 1785)

Haris, 1998

Macrophya blanda (Fabricius, 1775)

Böhönye, SMM

Macrophya crassula (Klug, 1817)

Haris, 1998

Macrophya duodecimpunctata (Linné, 1758)

Haris, 1998

Macrophya erythrocnema Costa, 1860

Haris, 1998

Macrophya militaris (Klug, 1817)

Haris, 1998

Macrophya montana (Scopoli, 1763)

Hanis, 1998 mint Maoophya nstia (Linné, 1758)

Macrophya postica (Brullé, 1832)

Haris, 1998 
Macrophya punctum-album (Linné)

Látrány, SMM

Macrophya recognata Zombori, 1979

Haris, 1998

Macrophya ribis (Schrank, 1781)

Haris, 1998

Macrophya rufipes (Linné, 1758)

Haris, 1998

Macrophya sanguinolenta (Gmelin, 1790)

Haris, 1998

Pachyprotasis antennata (Klug, 1817)

Haris, 1998

Pachyprotasis rapae (Linné, 1767)

Haris, 1998, Zombori, 1985

Rhogogaster scalaris (Klug, 1817)

Haris, 1998

Rhogogaster viridis (Linné, 1758)

Haris, 1998, Zombori, 1985

Sciapteryx consobrina (Klug, 1816)

Haris, 1998

Sciapteryx costalis (Fabricius, 1775)

Haris, 1998

Tenthredo amoena Gravenhorst, 1807

Haris, 1998

Tenthredo arcuata Förster, 1771

Haris, 1998

Tenthredo atra Linné, 1758

Haris, 1998

Tenthredo bifasciata rossi (Panzer, 1805)

Haris, 1998 mint Tenthredo rossi Panzer, 1805

Tenthredo campestris Linné, 1758

Haris, 1998

Tenthredo distinguenda Stein, 1885

Haris, 1998

Tenthredo excellens (Konow, 1886)

Haris, 1998

Tenthredo livida Linné, 1758

Haris, 1998

Tenthredo maculata Geoffroy, 1785

Haris, 1998

Tenthredo marginella Fabricius, 1793

Haris, 1998

Tenthredo mesomelas Linné, 1758

Haris, 1998

Tenthredo neobesa Zombori, 1980

Babócsa, SMM.

Tenthredo omissa (Förster, 1844)

Haris, 1998, Zombori, 1985

Tenthredo procera Klug, 1817

Potony, SMM

Tenthredo scrophulariae Linné, 1758

Haris, 1998

Tenthredo solitaria Scopoli, 1763

Zákány, SMM
Tenthredo temula Scopoli, 1763

Haris, 1998

Tenthredo vespa Retzius, 1783

Haris, 1998

Tenthredo zona Klug, 1817

Balatonszárszó, SMM

Tenthredo zonula Klug, 1817

Zombori, 1985

Tenthredopsis parida (Fabricius, 1775)

Haris, 1998

Tenthredopsis inomata Cameron, 1881

Haris, 1998

Tenthredopsis lactiflua (Klug, 1817)

Haris, 1998

Tenthredopsis litterata (Geoffroy, 1785)

Haris, 1998

Tenthredopsis nassata (Linné, 1767)

Haris, 1998

Tenthredopsis parvula Konow, 1890

Haris, 1998

Tenthredopsis sordida (Klug, 1817)

Haris, 1998

Tenthredopsis stigma (Fabricius, 1798)

Haris, 1998, Zombori, 1985

Tenthredopsis tarsata (Fabricius, 1804)

Haris, 1998

Tenthredopsis tesselata (Klug, 1817)

Haris, 1998, Zombori, 1985

\section{Nematinae}

Amauronematus lateralis Konow, 1896

Látrány, SMM

Amauronematus viduatus (Zetterstedt, 1838)

Látrány, SMM

Euura atra (Jurine, 1807)

Haris, 1998

Cladius difformis (Panzer, 1799)

Haris, 1998

Cladius pectinicornis (Geoffroy, 1785)

Vízvár, SMM

Croesus latipes (Villaret, 1832)

Haris, 1998

Croesus varus. (Villaret, 1832)

Haris, 1998

Hemichroa crocea (Geoffroy, 1785)

Haris, 1998

Hoplocampa craetegi (Klug, 1816)

Haris, 1998, Zombori, 1985

Hoplocampa chrysorrhoea (Klug, 1816)

Látrány, SMM.

Hoplocampa minuta (Christ, 1791)

Haris, 1998

Hoplocampa rutilicornis (Klug, 1816)

Zombori, 1982 
Mesoneura opaca (Klug, 1819)

Zombori, 1985

Nematinus luteus (Panzer, 1805)

Lálrány, SMM

Nematinus willigkiae Stein, 1926

Haris, I 998

Nematus bergmanni Dahlbom, 1835

Haris, 1998

Nematus bipartitus Lepeletier, 1823

Haris, 1998

Nematus melanaspis Hartig, I 840

Haris, 1998

Nematus myosotidis (Fabricius, 1804)

Haris, 1998

Nernatus oligospylus Förster, 1854

Haris, 1998

Nematus salicis (Linné, 1758)

Mocsáry, 1900 mint Pteronus salicis (Linné, 1758)

Nematus tibialis Newman, 1837

Haris, 1998

Nematus ribessii (Scopoli, 1763)

Zamárdi, MTM

Nematus silvestris Cameron

Kaposméró

Pachynematus clitellatus (Serville, 1823)

Haris, 1998

Pachynematus ragus (Fabricius, 178I)

Kis-Balaton, Lebujpuszta, MTM
Pachynematus xanthocarpus (Hartig, 1840)

Haris, 1998

Phyllocolpa leucaspis (Tischbein, 1846)

Látrány, SMM

Phyllocolpa leucosticta (Hartig, 1837)

Látrány, SMM

Pontania viminalis (Linné, 1758)

Balatonberény, MTM

Priophorus morio (Lepeletier, 1823)

Haris, 1998; Zombori, 1985 (=Priophorus

bruelli Dahlbom, 1835 néven)

Priophorus pallipes (Lepeletier, 1823)

Haris, 1998

Pristiphora aquilegiae (Vollenhoven, 1866)

Látrány, SMM

Pristiphora bifida Hellén, 1947

Somogymeggyes, SMM

Pristiphora conjugata (Dahlbom, 1835)

Haris, 1998

Pristiphora armata (Thomson, 1862)

Haris, 1998

Pristiphora melanocarpa (Hartig, 1840)

Haris, 1998

Pristiphora carinata (Hartig, 1837)

Haris, 1998

Pseudodineura fuscula (Klug, 1816)

Haris, 1998

\section{Értékelés}

Magyarország faunájára új fajok: Amauronematus lateralis Konow, 1896, Phyllocolpa leucaspis (Tischbein, 1846), Pristiphora bifida Hellén, 1947

Magyarországon csak Somogy megyéból ismert fajok: Abia fulgens Zaddach, 1863, Aprosthema bifida (Klug, 1834), Dolerus thoracicus (Fallén, 1808), Hemichroa crocea (Geoffroy, 1785), Pristiphora conjugata (Dahlbom, 1835), Pristiphora discoidalis (C. G. Thomson, 1871), Pristiphora armata (Thomson, 1862), Pristiphora carinata (Hartig, 1837).

Ritka fajok: Athalia paradoxa Konow, 1886, Dolerus megapterus Cameron, 1881, Dolerus quadrinotatus Bíró, 1884, Pamphilius marginatus (Lepeletier, 1823), Parna tenella (Klug, 1816), Strongylogaster xanthocera (Stephens, 1835), Tomostethus funereus (Klug, 1816), Tremex alchymista Mocsáry, I 836, Trichosoma vitellinae (Linné, 1761). 
1. táblázat: Somogyban ismert Symphyta fajok családonkénti megoszlása Table 1.: Families distribution of the Symphyta of Somogy county

\begin{tabular}{|l|c|}
\hline Családok & Fajok száma \\
\hline Xyelidae & 0 \\
\hline Pamphilidae & 6 \\
\hline Megalodontidae & 4 \\
\hline Blasticotomidade & 0 \\
\hline Argidae & 12 \\
\hline Cimbicidae & 10 \\
\hline Diprionidae & 5 \\
\hline Tenthredinidaø & 168 \\
\hline Siricidae & 2 \\
\hline Xiphidriidae & 2 \\
\hline Cephidae & 10 \\
\hline Orussidae & 1 \\
\hline
\end{tabular}

\section{Irodalom}

HArıs, A. 1998: A Somogy Megyei Múzeum levéldarázs-gyújteménye (Hymenoptera, Symphyta). Somogy Megyei Múzeumok Közleményei 13: 275 - 285.

MOCSÁRY, S. 1900: Hymenoptera. - In Mocsáry S. Fauna Regni Hungariae. 7-28.

MÓCZÁR, L. et ZOMBORI, L. (1973) Levéldarázs-alkatúak I. Tenthredinoidea I. - Fauna Hungariae 111 ., Akadémiai Kiadó, Budapest. 128 pp.

ZombOrı, L. 1982: Levéldarázs-alkatúak II. Tenthredinoidea II. - Fauna Hungariae 153., Akadémiai Kiadó, Budapest. 144 pp.

ZOMBOR, L. 1985: Adatok a Barcsi Borókás növényevő darazsainak ismeretéhez (Hymenoptera, Symphyta). - Dunántúli Dolgozatok Természettudományi Sorozat 5: 171 - 176.

ZOMBORI, L. 1990: Levéldarázs-alkatúak III. Tenthredinoidea III. - Fauna Hungariae 165. Akadémiai Kiadó, Budapest. 81 pp. 


\title{
Catalogue of the sawfly fauna of Somogy county (Hymenoptera: Symphyta)
}

\begin{abstract}
Atrila Haris
Altogether 220 species are listed from Somogy county which belong to 11 families. New records for the fauna of Hungary are: Amauronematus lateralis Konow, 1896, Phyllocolpa leucaspis (Tischbein, 1846), Pristiphora bifida Hellén, 1947.

These species are known only from Somogy county in Hungary: Abia fulgens Zaddach, 1863, Aprosthema bifida (Klug, 1834), Dolerus thoracicus (Fallén, 1808), Hemichroa crocea (Geoffroy, 1785), Pristiphora conjugata (Dahlbom, 1835), Pristiphora discoidalis (C. G. Thomson, 1871), Pristiphora armata (Thomson, 1862), Pristiphora carinata (Hartig, 1837).

Futher rare species are: Athalia paradoxa Konow, 1886, Dolerus megapterus Cameron, 1881, Dolerus quadrinotatus Bíró, 1884, Pamphilius marginatus (Lepeletier, 1823), Parna tenella (Klug, 1816), Strongylogaster xanthocera (Stephens, 1835), Tomostethus funereus (Klug, 1816), Tremex alchymista Mocsáry, 1836, Trichosoma vitellinae (Linné, 1761).
\end{abstract}

Address of correspondes:

Dr. Attila HARIS

Hungarian National History Museum

$\mathrm{H}-1088$ Budapest

Baross u 13.

HUNGARY 